\title{
Optically-guided frameless linac-based radiosurgery for brain metastases: clinical experience
}

\author{
Sameer K. Nath · Joshua D. Lawson · Jia-Zhu Wang • \\ Daniel R. Simpson · C. Benjamin Newman • \\ John F. Alksne · Arno J. Mundt · Kevin T. Murphy
}

Received: 1 June 2009/Accepted: 9 August 2009/Published online: 23 August 2009

(C) The Author(s) 2009. This article is published with open access at Springerlink.com

\begin{abstract}
The purpose of this study was to describe our clinical experience using optically-guided linear accelerator (linac)-based frameless stereotactic radiosurgery (SRS) for the treatment of brain metastases. Sixty-five patients (204 lesions) were treated between 2005 and 2008 with frameless SRS using an optically-guided bite-block system. Patients had a median of 2 lesions (range, 1-13). Prescription dose ranged from 14 to $22 \mathrm{~Gy}$ (median, $18 \mathrm{~Gy}$ ) and was given in a single fraction. Clinical and radiographic evaluation occurred every 2-4 months following treatment. At a median follow-up of 6.2 months, actuarial survival at 12 months was $40 \%$ [95\% confidence interval (CI), 28-52). Of 135 lesions that were evaluable for local control (LC), 119 lesions (88\%) did not show evidence of progression. Actuarial 12 month LC was $76 \%$ (95\% CI, 66-86). Tumors $\leq 2 \mathrm{~cm}$ in size had a better 12 month LC rate $(81 \%$ vs. $36 \%, P=0.017)$ than those $>2 \mathrm{~cm}$. Adverse events occurred in three patients $(5 \%)$. Optically-guided
\end{abstract}

Abstract will be presented at the 2009 A.S.T.R.O. annual meeting.

S. K. Nath $(\bowtie)$. J. D. Lawson · J.-Z. Wang ·

D. R. Simpson - A. J. Mundt · K. T. Murphy

Department of Radiation Oncology, Rebecca and John Moores

Comprehensive Cancer Center, University of California San

Diego, 3855 Health Sciences Dr. \#0843, La Jolla,

CA 92093-0843, USA

e-mail: sameerknath@gmail.com

C. B. Newman · J. F. Alksne

Division of Neurosurgery, Department of Surgery, Rebecca and John Moores Comprehensive Cancer Center, University of California San Diego, La Jolla, CA, USA

S. K. Nath - J. D. Lawson - D. R. Simpson · A. J. Mundt Center for Advanced Radiotherapy Technologies, Rebecca and John Moores Comprehensive Cancer Center, University of California San Diego, La Jolla, CA, USA linac-based frameless SRS can produce clinical outcomes that compare favorably to frame-based techniques. As this technique is convenient to use and allows for the uncomplicated delivery of hypofractionated radiotherapy, frameless SRS will likely have an increasingly important role in the management of brain metastases.

Keywords Frameless - Stereotactic radiosurgery · Optically-guided $\cdot$ Brain metastases

\section{Introduction}

Stereotactic radiosurgery (SRS) has a well-established role for the treatment of intracranial disease and has been used specifically for the management of brain metastases in the United States since the mid 1980s [1]. The efficacy of SRS for the treatment of brain metastases has been demonstrated in several randomized trials and multi-institutional studies [2-5].

Conventionally, SRS is performed with the use of a stereotactic head frame that is affixed to the calvarium in order to provide rigid patient immobilization during planning and treatment delivery. The use of a head frame in SRS has been extensively studied and has been shown to be associated with excellent target localization during both planning and treatment delivery $[6,7]$. However, although this system provides a high-degree of accuracy that is necessary when using large and highly-conformal doses, there are several disadvantages of frame-based immobilization, including patient discomfort, difficulty performing hypofractionated therapy, and additional effort required to coordinate between personnel on different services.

As an alternative to frame-based technologies, there is now a variety of frameless systems that have been devised 
using both CyberKnife ${ }^{\circledR}$ (Accuray Inc., Sunnyvale, CA) and traditional linear accelerator (linac)-based platforms. These systems differ in their methods for patient tracking, as well as their ability to perform various types of modern radiotherapy advancements, such as image-guided radiotherapy (IGRT) and intensity-modulated SRS (IMSRS). However, only limited clinical data on the use of these systems for intracranial metastatic disease has been reported [8-12]. Moreover, even fewer studies have provided data specifically on linac-based frameless devices $[10,11]$. As the ultimate validation of a new procedure is measured in terms of clinical outcomes, it is important to provide treatment data from actual patients prior to widespread acceptance.

In 2005, the University of California at San Diego adopted an optically-guided linac-based frameless SRS system (Varian Triology, Varian Oncology Systems, Palo Alto, CA), which has been used to treat over 200 patients with either metastatic or primary CNS disease. In this paper, we describe our clinical experience using opticallyguided frameless SRS to manage brain metastases.

\section{Materials and methods}

Patient selection

All patients were treated at the University of California at San Diego in the Rebecca and John Moores Comprehensive Cancer Center between December 2005 and June 2008 using frameless SRS. Patients with intracranial metastatic disease that was histologically verified at either the primary or metastatic site were selected for treatment if they were not surgical candidates and were able to lie still and tolerate simulation. Initially, patients with greater than four metastases were offered frameless SRS only if they had refused whole-brain radiotherapy (WBRT), however we transitioned to treating patients with greater than four metastases after our initial clinical experiences were encouraging.

Patient and treatment characteristics

A total of 65 patients with 204 lesions were treated for intracranial metastatic disease. Patient characteristics are summarized in Table 1. The largest tumor diameter was measured for each lesion, and the median value was $9 \mathrm{~mm}$ (range, 1-35 mm). Prescription dose was 14-22 Gy (median, $18 \mathrm{~Gy}$ ) and was given in a single fraction. Specific doses were determined by the RTOG 90-05 report [13]. Radiation therapy was delivered in rotational arc beams with circular cone collimation in 37 patients $(57 \%)$ who had both small and few (three or fewer) lesions. For patients with either a large and irregular tumor or with
Table 1 Patient characteristics

\begin{tabular}{|c|c|}
\hline & Number $(\%)$ \\
\hline Total patients & 65 \\
\hline Total lesions & 204 \\
\hline \multicolumn{2}{|l|}{ Sex } \\
\hline Male & $27(41)$ \\
\hline Female & $38(59)$ \\
\hline \multicolumn{2}{|l|}{ Age } \\
\hline Median & 58 \\
\hline Range & $20-83$ \\
\hline \multicolumn{2}{|l|}{ Primary tumor } \\
\hline Lung & $28(44)$ \\
\hline Breast & $20(31)$ \\
\hline Melanoma & $12(18)$ \\
\hline Ovarian & $2(3)$ \\
\hline Other & $3(4)$ \\
\hline \multicolumn{2}{|c|}{ Number of isocenters } \\
\hline Median & 1 \\
\hline Range & $1-5$ \\
\hline \multicolumn{2}{|l|}{ Dose (Gy) } \\
\hline Median & 18 \\
\hline Range & $14-22$ \\
\hline \multicolumn{2}{|c|}{ Lesions per patient } \\
\hline Median & 2 \\
\hline Range & $1-13$ \\
\hline
\end{tabular}

multiple lesions (28 patients, $43 \%$ ), IMSRS with a dynamic multi-leaf collimator (MLC) was used. Treatment characteristics are summarized in Table 2. Frameless SRS was performed with upfront WBRT in 10 patients (15\%). Salvage therapy was offered to patients with recurrent local disease or new intracranial metastases and consisted of repeated frameless SRS, WBRT or surgery.

Frameless radiosurgery procedure

After obtaining informed consent, patients underwent contrast-enhanced T1-weighted magnetic resonance imaging (MRI) $(26 \mathrm{~cm}$ FOV, $512 \times 512$ pixel size, $1.5 \mathrm{~mm}$ slice intervals) using a 3.0 Tesla MRI (General Electric, Fairfield, CT). Subsequently, customized immobilization was then designed for each patient and consisted of an Accuform ${ }^{\mathrm{TM}}$ (CIVCO Medical Solutions, Kalona, IA) headrest fitted to the patient's head in neutral position, a molded thermoplastic face mask, and a customized bite block.

After the bite-block had been fashioned, the patient was taken to the treatment vault for verification. In the vault, the patient was fitted with a head band that had an attached set of reference markers. The bite-block was then removed and replaced 10 times and the positioning error was measured each time through the optical-guidance apparatus. If 
Table 2 Treatment characteristics

\begin{tabular}{lc}
\hline Treatment type & Number of Pts $(\%)$ \\
\hline SRS alone & $53(81.5)$ \\
SRS + WBRT & $9(14.0)$ \\
$\mathrm{Sx}+\mathrm{SRS}$ & $1(1.5)$ \\
$\mathrm{Br}+\mathrm{SRS}$ & $1(1.5)$ \\
$\mathrm{Br}+\mathrm{SRS}+$ WBRT & $1(1.5)$ \\
Total & $65(100)$ \\
\hline
\end{tabular}

Pts patients, $S x$ surgery, $B r$ intracavitary brachytherapy, $S R S$ stereotactic radiosurgery, WBRT whole brain radiotherapy

the variation between all 10 trials was less than $0.75 \mathrm{~mm}$, the bite block was accepted for use.

Patients were then simulated supine with the thermoplastic mask, headrest, and bite block in place using noncontrast brain computed tomography (CT) $(35 \mathrm{~cm}$ FOV, $512 \times 512$ pixel size, $1.25 \mathrm{~mm}$ slice intervals). An initial review of the $\mathrm{CT}$ was performed to ensure that all fiducial markers were visible and that no significant motion abnormality occurred. The MRI and CT were then transferred to the planning center and fused using a rigid autoregistration tool and subsequently manually verified.

Planning was performed using Varian FastPlan ${ }^{\circledR}$ software for cone-based plans or Varian Eclipse ${ }^{\mathrm{TM}}$ software for IMSRS plans and was based on the enhancing tumor volume with an additional 1-2 $\mathrm{mm}$ margin. Single and multiple isocenter plans were constructed for one or more lesions under the guidance of the radiation oncologist, neurosurgeon, and medical physicist. The $80 \%$ isodose curve was selected for each patient planned with conebased collimation, and approximately the $90 \%$ isodose line was selected for IMSRS patients.

On treatment day, patient setup and isocenter localization were performed with the optical-guidance system. In addition, a pair of orthogonal kilovoltage $(\mathrm{kV})$ images were taken and compared to digitally reconstructed radiographs (DRRs) generated from the planning CT. These images were used for verification purposes only and were not used for repositioning. Radiation was generated from a Varian Trilogy linear accelerator (linac) using the $6 \mathrm{MV}$ radiosurgery mode. Treatment times were generally less than $45 \mathrm{~min}$ or up to $1 \mathrm{~h}$ if including patient setup time.

Follow-up, statistical analysis and toxicity

Patients were routinely seen 1 week following SRS for a clinical exam. Contrast-enhanced MRI and physician evaluation was performed every 3 months until 18 months, and then routine surveillance was determined on a case-bycase basis. Treatment response was analyzed by survival and local control. Local control was defined as the absence of progression; progression was defined as an increase of greater than $20 \%$ of the sum of the two largest diameters of the lesion. Intracranial status was deemed as unknown if at least one follow-up imaging exam was not performed. These patients were excluded from local control data, however they were included in survival analysis. Of those with radiographic follow-up, imaging included MRI (92\%) and CT (8\%). Each MRI was independently evaluated for progression by a radiologist, a radiation oncologist, and a neurosurgeon. Elsewhere-brain failure was defined as new intracranial metastatic disease occurring outside of the treatment volume on radiographic examination, and regional control was defined as the absence of elsewherebrain failure on follow-up imaging. Survival, local failure, and regional control were estimated by the Kaplan-Meier method. All statistical analyses were performed using NCSS (originally, Number Cruncher Statistical System) (NCSS LLC, Kaysville, UT). Toxicity was graded according to the Radiation Therapy Oncology Group (RTOG) scale [14].

\section{Results}

The median follow-up for all patients was 6.2 months (range, 0.13-32.4). Sixteen were alive at the time of analysis and had a median follow-up of 18.1 months (range 7.5-32.4). The 12-month actuarial survival for the entire group was $40 \%$ [95\% confidence interval (CI), 28-52\%) (Fig. 1). No statistically significant difference in survival between patients treated with frameless SRS alone or combined with WBRT was detected (12-month actuarial survival $39 \%$ vs. $46 \%, P=0.40$ ).

Seventeen patients were excluded from local control and regional control analysis owing to unknown intracranial status at the time of death. Of the 48 remaining patients with 135 lesions who were radiographically evaluable for local control, 37 patients (77\%) and 119 lesions (88\%) did

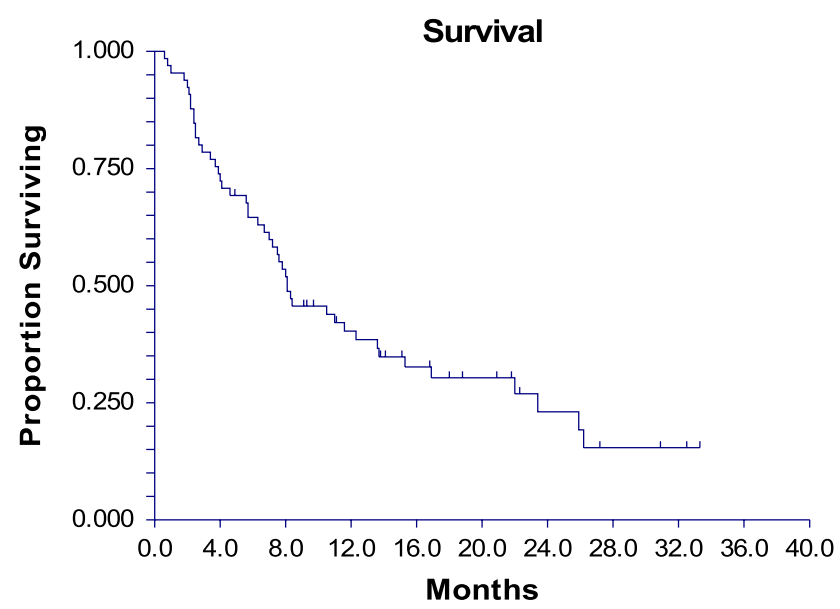

Fig. 1 Overall survival for all patients treated with frameless SRS 


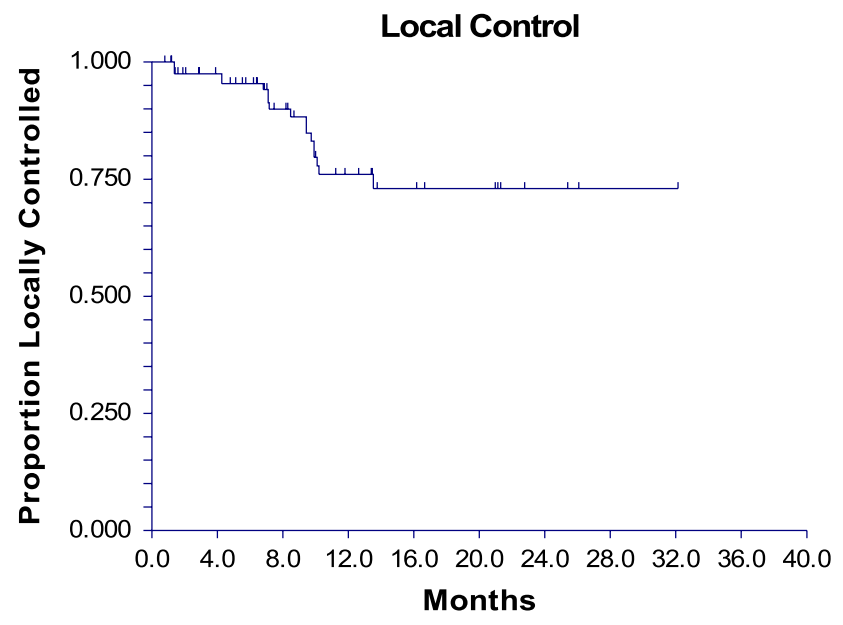

Fig. 2 Local control for patients treated with frameless SRS

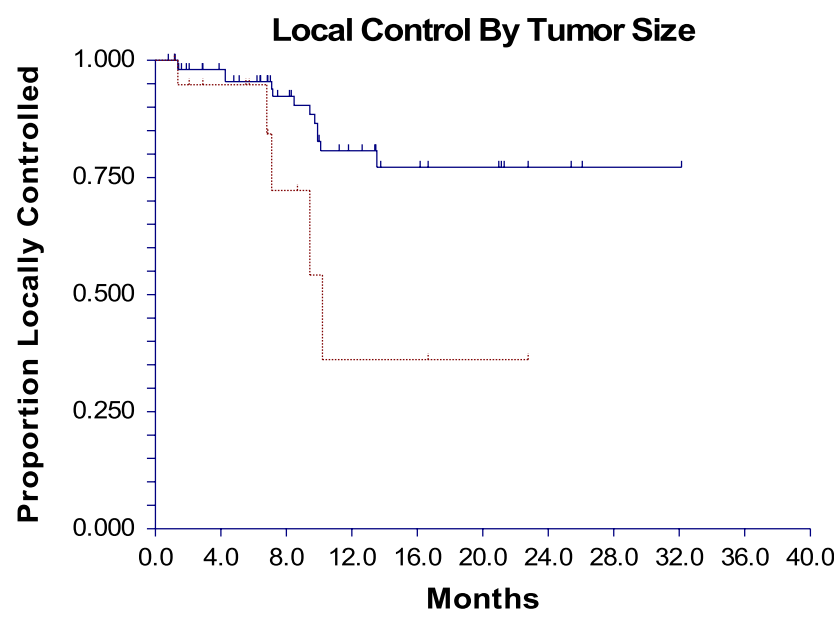

Fig. 3 Local control stratified by tumors $\leq 2 \mathrm{~cm}$ (solid line) and $>2 \mathrm{~cm}$ (dashed line)

not show evidence of progression. Actuarial 12 month local control was 76\% (95\% CI, 66-86\%) (Fig. 2). Tumors $\leq 2 \mathrm{~cm}$ in size had a better 12 month local control rate than those $>2 \mathrm{~cm}(81 \%$ vs. $36 \%, P=0.017)$ (Fig. 3). No statistically significant difference was observed between lesions treated with and without WBRT $(P=0.31)$.

Elsewhere-brain failures were detected in 23 patients (48\%). Actuarial 12 month regional control was 46\% (95\% CI, 30-62\%) (Fig. 4). No statistically significant difference in regional control was observed between patients treated with and without WBRT $(P=0.83)$. Salvage therapy was performed for patients with local failures or new intracranial metastatic disease and consisted of repeat SRS (nine patients, 14\%), WBRT (seven patients, 11\%), repeat SRS and WBRT (three patients, 5\%), and surgery (two patients, $3 \%$ ). Of patients treated with repeat SRS, nine patients received a single re-treatment, two patients received two re-treatments, and one patient received four re-treatments.
Regional Control

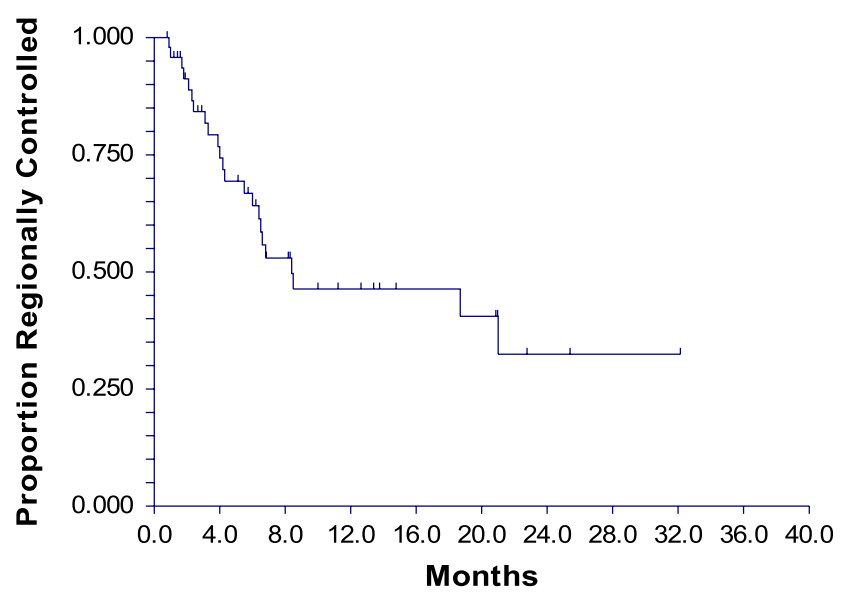

Fig. 4 Regional control for patients treated with frameless SRS

Nine patients (14\%) had grade 2 toxicities; one patient had a single seizure, one patient had transient mild ataxia, and seven had edema-associated symptoms that resolved with steroids. Grade 3 or higher toxicities occurred in three patients (5\%); one patient developed aphasia, one patient with metastatic melanoma developed hemorrhage in a treated lesion, and one patient developed hemiparesis secondary to radionecrosis and required surgery.

\section{Discussion}

Frameless SRS is a relatively new treatment that avoids the use of invasive head frames for patient immobilization and provides several distinct advantages over frame-based techniques. Although several systems are currently in use, limited clinical data exist to demonstrate the safety and efficacy of frameless devices, and data are especially lacking on systems that employ conventional linac-based platforms. In this study, we present encouraging clinical outcomes on a series of patients treated with opticallyguided frameless linac-based SRS for the management of brain metastases.

Local control was selected as a primary endpoint, as it is a sensitive clinical outcome for assessing targeting accuracy. Frame-based SRS has been shown to produce 12-month actuarial local-control rates ranging from 64 to $89 \%$ (Table 3) [4, 15-21]. Our actuarial local control rate was $76 \%$ at 12 months, which compares favorably to frame-based, as well as other frameless SRS techniques (Table 3). Furthermore, a unique advantage of the present series of patients is that the majority of our patients $(82 \%)$ did not receive upfront WBRT or surgery, and therefore the influence of WBRT and other initial therapies on our local control rate was minimized. In contrast, other frameless 
Table 3 Comparison of survival and local control rates between frame-based and frameless SRS in non-randomized studies on brain metastases

\begin{tabular}{|c|c|c|c|c|c|}
\hline Study (year) & Pts & Treatment system & Crude LC (\%) & Actuarial 1-year LC (\%) & Actuarial 1-year survival (\%) \\
\hline \multicolumn{6}{|l|}{ Frame-based SRS } \\
\hline Becker [15] & 55 & Linac-based & 92 & 61 & 33 \\
\hline Sneed [4] & 268 & Gamma Knife $e^{\mathrm{a}}$ & - & - & 38 \\
\hline Chitapanarux [16] & 41 & Linac-based & $76^{\mathrm{b}}$ & 68 & 48 \\
\hline Datta [17] & 53 & Gamma Knife & 89 & - & 23 \\
\hline Schomas [19] & 80 & Linac-based & 91 & 89 & 33 \\
\hline Bhatnagar [20] & 205 & Gamma Knife & - & 71 & $37^{\mathrm{c}}$ \\
\hline Rades [18] & 94 & Linac-based $^{\mathrm{d}}$ & - & 64 & 54 \\
\hline Serizawa [21] & 2,390 & Gamma Knife & - & - & $30^{\mathrm{c}}$ \\
\hline \multicolumn{6}{|l|}{ Frameless SRS } \\
\hline Shimamoto [8] & 41 & CyberKnife ${ }^{\circledR}$ & - & $80^{\mathrm{e}}$ & $26^{\mathrm{f}}$ \\
\hline Kamath [10] & 64 & Linac-based & $88^{\mathrm{b}}$ & $40^{\mathrm{c}, \mathrm{g}}$ & $38^{\mathrm{c}}$ \\
\hline Nishizaki [9] & 71 & CyberKnife ${ }^{\circledR}$ & 83 & - & 47 \\
\hline Breneman [11] & 53 & Linac-based & - & 80 & 44 \\
\hline Nath (present series) & 65 & Linac-based & 88 & 76 & 40 \\
\hline
\end{tabular}

- , not reported

Pts patients, $L C$ local control

a $76 \%$ Gamma Knife and $24 \%$ linac-based

b By patient

${ }^{c}$ Estimated from Kaplan-Meier curve

d $71 \%$ linac-based and $29 \%$ Gamma Knife

e Freedom from progression for patients treated with $\geq 24$ Gy

${ }^{\mathrm{f}}$ For patients treated with $\geq 24$ Gy

g Local control determined by patient

studies have reported only $26-40 \%[10,11]$ of patients treated with frameless SRS alone.

Survival was also analyzed as an endpoint. Frame-based SRS 12-month actuarial survival rates range from 23 to $54 \%$ (Table 3). Our experience with frameless SRS resulted in a 12 month actuarial survival rate of $40 \%$, which also compares favorably to both frame-based, as well as other frameless SRS techniques (Table 3).

Frameless techniques have several advantages over frame-based approaches, including added patient comfort, convenience of use, and improved ability to perform hypofractionated therapy for large lesions. Generally, SRS is recommended for lesions less than $3 \mathrm{~cm}$ [22]. However, research has suggested that patients may benefit from hypofractionated SRS for larger tumors [9, 23]. Although hypofractionation is possible with frame-based systems, frameless systems can offer improved patient comfort and less complicated delivery of therapy.

Clinical outcomes have now been reported on each of the frameless radiosurgery systems currently available. CyberKnife $^{\circledR}$ offers a frameless system that employs a compact linac mounted on a robotic-arm that acquires X-ray based images for precise target localization [24]. Novalis $^{\mathrm{TM}}$
(BrainLab Inc, Chicago, IL) offers a system that uses both optical-guidance of reflective fiducials placed over the patient's mask for initial positioning and orthogonal kilovoltage images for added image-guidance [11]. RadioCameras (Zmed, Ashland, MA) provides a linac-based system that employs an optically-guided bite-block [10], which was later adapted by Varian and combined with the Trilogy ${ }^{\mathrm{TM}}$ machine to allow for high-output delivery along with IGRT and IMSRS. Clinical outcomes reported by these systems are similar (Table 3), and the advantages of each technique depend on various institutional preferences.

Although these results are promising, our analysis has several limitations. First, this is a retrospective study and thus suffers from the inherent limitations of such an analysis. Second, not all patients underwent follow-up imaging to document local control, and therefore, local failures may have been missed. Unfortunately, this is a common problem with research on brain metastases, as many patients die within a few months of treatment.

In conclusion, we report on our clinical experience using an optically-guided linac-based frameless SRS system for the treatment of brain metastases. Our results show encouraging clinical outcomes that compare favorably to 
traditional frame-based as well as other frameless technologies and support the continued role of frameless SRS in the management of patients with brain metastases.

Acknowledgments This research was supported by an NIH T32 training grant (RR023254). Conflicts of interest statement A.J.M and K.T.M have received honorariums from Varian Medical Systems for giving lectures on varying topics in radiotherapy. The Center for Advanced Radiotherapy Technologies receives funding from Varian Medical Systems for research unrelated to the current project.

Open Access This article is distributed under the terms of the Creative Commons Attribution Noncommercial License which permits any noncommercial use, distribution, and reproduction in any medium, provided the original author(s) and source are credited.

\section{References}

1. Lunsford LD, Flickinger J, Lindner G, Maitz A (1989) Stereotactic radiosurgery of the brain using the first United States 201 cobalt-60 source gamma knife. Neurosurgery 24:151-159

2. Kondziolka D, Patel A, Lunsford LD, Kassam A, Flickinger JC (1999) Stereotactic radiosurgery plus whole brain radiotherapy versus radiotherapy alone for patients with multiple brain metastases. Int J Radiat Oncol Biol Phys 45:427-434

3. Andrews DW, Scott CB, Sperduto PW, Flanders AE, Gaspar LE, Schell MC, Werner-Wasik M, Demas W, Ryu J, Bahary JP, Souhami L, Rotman M, Mehta MP, Curran WJ Jr (2004) Whole brain radiation therapy with or without stereotactic radiosurgery boost for patients with one to three brain metastases: phase III results of the RTOG 9508 randomised trial. Lancet 363: $1665-1672$

4. Sneed PK, Suh JH, Goetsch SJ, Sanghavi SN, Chappell R, Buatti JM, Regine WF, Weltman E, King VJ, Breneman JC, Sperduto PW, Mehta MP (2002) A multi-institutional review of radiosurgery alone vs. radiosurgery with whole brain radiotherapy as the initial management of brain metastases. Int J Radiat Oncol Biol Phys 53:519-526

5. Aoyama H, Shirato H, Tago M, Nakagawa K, Toyoda T, Hatano K, Kenjyo M, Oya N, Hirota S, Shioura H, Kunieda E, Inomata T, Hayakawa K, Katoh N, Kobashi G (2006) Stereotactic radiosurgery plus whole-brain radiation therapy vs stereotactic radiosurgery alone for treatment of brain metastases: a randomized controlled trial. JAMA 295:2483-2491

6. Yeung D, Palta J, Fontanesi J, Kun L (1994) Systematic analysis of errors in target localization and treatment delivery in stereotactic radiosurgery (SRS). Int J Radiat Oncol Biol Phys 28: 493-498

7. Kitchen ND, Lemieux L, Thomas DG (1993) Accuracy in framebased and frameless stereotaxy. Stereotact Funct Neurosurg 61:195-206

8. Shimamoto S, Inoue T, Shiomi H, Sumida I, Yamada Y, Tanaka E (2002) CyberKnife stereotactic irradiation for metastatic brain tumors. Radiat Med 20:299-304

9. Nishizaki T, Saito K, Jimi Y, Harada N, Kajiwara K, Nomura S, Ishihara H, Yoshikawa K, Yoneda H, Suzuki M, Gibbs IC (2006) The role of cyberknife radiosurgery/radiotherapy for brain metastases of multiple or large-size tumors. Minim Invasive Neurosurg 49:203-209
10. Kamath R, Ryken TC, Meeks SL, Pennington EC, Ritchie J, Buatti JM (2005) Initial clinical experience with frameless radiosurgery for patients with intracranial metastases. Int J Radiat Oncol Biol Phys 61:1467-1472

11. Breneman JC, Steinmetz R, Smith A, Lamba M, Warnick RE (2009) Frameless image-guided intracranial stereotactic radiosurgery: clinical outcomes for brain metastases. Int J Radiat Oncol Biol Phys

12. Furuse M, Aoki T, Takagi T, Takahashi JA, Ishikawa M (2008) Frameless stereotactic radiosurgery with a bite-plate: our experience with brain metastases. Minim Invasive Neurosurg 51: 333-335

13. Shaw E, Scott C, Souhami L, Dinapoli R, Kline R, Loeffler J, Farnan N (2000) Single dose radiosurgical treatment of recurrent previously irradiated primary brain tumors and brain metastases: final report of RTOG protocol 90-05. Int J Radiat Oncol Biol Phys 47:291-298

14. Cox JD, Stetz J, Pajak TF (1995) Toxicity criteria of the radiation therapy oncology group (RTOG) and the European organization for research and treatment of cancer (EORTC). Int J Radiat Oncol Biol Phys 31:1341-1346

15. Becker G, Jeremic B, Engel C, Buchgeister M, Paulsen F, Duffner F, Meisner C, Bamberg M (2002) Radiosurgery for brain metastases: the Tuebingen experience. Radiother Oncol 62:233-237

16. Chitapanarux I, Goss B, Vongtama R, Frighetto L, De Salles A, Selch M, Duick M, Solberg T, Wallace R, Cabatan-Awang C, Ford J (2003) Prospective study of stereotactic radiosurgery without whole brain radiotherapy in patients with four or less brain metastases: incidence of intracranial progression and salvage radiotherapy. J Neurooncol 61:143-149

17. Datta R, Jawahar A, Ampil FL, Shi R, Nanda A, D'Agostino H (2004) Survival in relation to radiotherapeutic modality for brain metastasis: whole brain irradiation vs. gamma knife radiosurgery. Am J Clin Oncol 27:420-424

18. Rades D, Bohlen G, Pluemer A, Veninga T, Hanssens P, Dunst J, Schild SE (2007) Stereotactic radiosurgery alone versus resection plus whole-brain radiotherapy for 1 or 2 brain metastases in recursive partitioning analysis class 1 and 2 patients. Cancer 109:2515-2521

19. Schomas DA, Roeske JC, MacDonald RL, Sweeney PJ, Mehta N, Mundt AJ (2005) Predictors of tumor control in patients treated with linac-based stereotactic radiosurgery for metastatic disease to the brain. Am J Clin Oncol 28:180-187

20. Bhatnagar AK, Flickinger JC, Kondziolka D, Lunsford LD (2006) Stereotactic radiosurgery for four or more intracranial metastases. Int J Radiat Oncol Biol Phys 64:898-903

21. Serizawa T, Yamamoto M, Nagano O, Higuchi Y, Matsuda S, Ono J, Iwadate Y, Saeki N (2008) Gamma Knife surgery for metastatic brain tumors. J Neurosurg 109(Suppl):118-121

22. Alexander EIII, Moriarty TM, Davis RB, Wen PY, Fine HA, Black PM, Kooy HM, Loeffler JS (1995) Stereotactic radiosurgery for the definitive, noninvasive treatment of brain metastases. J Natl Cancer Inst 87:34-40

23. Davey P, Schwartz ML, Scora D, Gardner S, O'Brien PF (2007) Fractionated (split dose) radiosurgery in patients with recurrent brain metastases: implications for survival. $\mathrm{Br} \mathrm{J}$ Neurosurg 21:491-495

24. Adler JR Jr, Chang SD, Murphy MJ, Doty J, Geis P, Hancock SL (1997) The Cyberknife: a frameless robotic system for radiosurgery. Stereotact Funct Neurosurg 69:124-128 